\title{
MULTI-ATOMIC YOUNG MEASURE AND ARTIFICIAL BOUNDARY IN APPROXIMATION OF MICROMAGNETICS
}

\author{
ZHIPING LI $^{1} \quad$ XIAONAN WU ${ }^{2}$ \\ ${ }^{1}$ LMAM, SCHOOL OF MATHEMATICAL SCIENCES, PEKING UNIVERSITY, \\ BEIJING 100871, CHINA \\ 2 DEPARTMENT OF MATHEMATICS, HONG KONG BAPTIST UNIVERSITY, \\ KOWLOON TONG, HONG KONG, CHINA
}

\begin{abstract}
Some micromagnetic phenomena can be modelled by a minimization problem of a nonconvex energy. A numerical method to compute the micromagnetic field, which gives rise to a finite dimensional unconstrained minimization problem, is given and analyzed. In our method, the Maxwell's equation defined on the whole space is solved by a finite element method using artificial boundary, and the highly oscillatory magnetization structure is approximated by an element-wise constant Young measure supported on a finite number of unknown points on the unit sphere. Numerical experiments on some uniaxial and cubic anisotropic energy densities show that the method is efficient.
\end{abstract}

\section{INTRODUCTION}

Some micromagnetic phenomena can be modelled by a minimization problem of a nonconvex energy $[1,2]$

$$
E(\mathbf{m})=\int_{\Omega} \varphi(\mathbf{m}) d x-\int_{\Omega} \mathbf{H} \cdot \mathbf{m} d x+\frac{1}{2} \int_{R^{n}}\left|\nabla u_{\mathbf{m}}\right|^{2} d x,
$$

where $\varphi: R^{n} \rightarrow R^{1}$, with $n=2$ or 3 , is a non-negative continuous function depending on material properties, $\mathbf{m}: \Omega \rightarrow R^{n}$ is an induced magnetic field satisfying $|\mathbf{m}|=1$ almost everywhere in $\Omega, \mathbf{H}$ is an applied external magnetic

1991 Mathematics Subject Classification. 49K20, 65K10, 65N15, 65N30, 65N50.

Key words and phrases. non-convex energy minimization, micromagnetic, microstructure, Young measure, unbounded domain, artificial boundary, finite element method.

The research was supported in part by the Special Funds for Major State Basic Research Projects (G1999032804), RFDP of China, and the RGC grant from Hong Kong. 
field, and $u_{\mathbf{m}}: R^{n} \rightarrow R^{1}$ is the potential of the induced magnetic field satisfying the Maxwell's equation

$$
\operatorname{div}\left(-\nabla u_{\mathbf{m}}+\mathbf{m} \chi_{\Omega}\right)=0, \quad \text { in } H^{-1}\left(R^{n}\right) .
$$

Here, $\chi_{\Omega}$ stands for the characteristic function of $\Omega$. It is well known that the above nonconvex variational problem typically can not attain the infimal energy in the Lebesgue-type functions and the minimizing sequences of the problem can develop microstructures, i.e. finer and finer highly oscillatory magnetization structures [3]. However, if the measure valued solutions are allowed, the relaxed problem is well posed $[4,5,6,7,8,9]$. Denote $\nu=\left\{\nu_{x}\right\}_{x \in \Omega}$ a family of probability measure supported on $S^{n-1}$ which is weakly measurable, i.e., $x \rightarrow \int_{S^{n-1}} v(A) \nu_{x}(d A)$ is measurable for any $v \in C\left(S^{n-1}\right)$. Let

$$
\mathfrak{A}=\left\{\nu=\left\{\nu_{x}\right\}_{x \in \Omega}: \operatorname{supp} \nu_{x} \subset S^{n-1} \text {, a.e. } x \in \Omega\right\},
$$

and, for any $\nu \in \mathfrak{A}$, let $\mathbf{m}(x)=\int_{S^{n-1}} A \nu_{x}(d A)$, for almost all $x \in \Omega$, let $u_{\mathbf{m}}$ be the solution to the Maxwell's equation (1.2), and define a relaxed energy functional by

$$
R E(\nu)=\int_{\Omega} \int_{S^{n-1}} \varphi(A) \nu_{x}(d A) d x-\int_{\Omega} \mathbf{H} \cdot \mathbf{m} d x+\frac{1}{2} \int_{R^{n}}\left|\nabla u_{\mathbf{m}}\right|^{2} d x .
$$

Then, the Young measure version of the relaxed problem of micromagnetics is to minimize the relaxed energy $R E(\cdot)$ in the set $\mathfrak{A}[5,8,9]$.

Numerical methods have been developed to solve the above relaxed problem. Kružik [10] used an element-wise constant three-atomic Young measures for a regular triangulation of $\Omega \subset R^{n}$. Kružik and Prohl [9] used an elementwise constant multi-atomic Young measures of prescribed support according to a triangulation of $S^{n-1}$ with an adaptive strategy which singles out the socalled active atoms. In these methods, the dependence of $u_{\mathbf{m}}$ on $\mathbf{m}$ through the Maxwell's equation on the whole space $R^{n}$ (see (1.2)) is explicitly given by the Green's formula [11], which, after the finite element discretization of $\Omega$, is given by a summation of itergations defined on every element of the regular triangulation of $\Omega$.

In the present paper, we propose a new numerical method. In our method we still use an element-wise constant multi-atomic Young measure for the Young measure approximation, however, motivated by the fact that there is always a Young measure $\nu \in \mathfrak{A}$ to the relaxed problem such that, for almost 
all $x \in \Omega$, the measure $\nu_{x}$ is supported on a limited number of points, in fact the number can be as low as $n+1$ in general $[4,9]$ and the maximum number can be estimated by the number of minima of the anisotropic energy density, and by the idea of the mesh transformation method [12, 13, 14], the support of our multi-atomic Young measure is limited to a fixed number of points on $S^{n-1}$ which, instead of being prescribed, are taken as a part of unknown to be decided in the optimization process. For the numerical solution of the Maxwell's equation (1.2), an artificial boundary with properly imposed (either exact or approximate) boundary conditions (see for instance $[16,17,18,19$, 20] among many others) is introduced to transform the unbounded domain problem into a bounded domain problem, and then a standard conforming finite element method [21] is applied to obtain a numerical solution of $u_{\mathbf{m}}$. By using the polar coordinates for the unit sphere $S^{n-1}$ and by writing the volume fractions in a unconstrained form in a similar way as in [15], the discrete relaxed problem can then be expressed in a form of a finite dimensional unconstrained nonconvex optimization problem.

We notice here that, as is shown in [22], the use of piecewise constant finite element magnetisation $\mathbf{m}$ and piecewise affine conforming finite element potential $u$ can cause instability of the discrete problem, especially when the external magnetic field is small. The main reasons are that the set $\{\operatorname{div} \mathbf{m}=$ 0 , in the discrete sense $\}$ is not empty and consists of pairs of oscillations, and the additional oscillations between the minimum points of the anisotropic energy density do not increase the anisotropic energy $\int_{\Omega} \int_{S^{n-1}} \varphi(A) \nu_{x}(d A) d x$. To overcome this difficulty, we add an extra term $\lambda \int_{\Omega}|\mathbf{m}-\mathbf{H}|^{2} d x$, where $\lambda>0$ is a small parameter, to the energy functional in computation to suppress the element level oscillations. To further reduce the artificial oscillations in the numerical result, we use the local average of $\mathbf{m}$ instead of $\mathbf{m}$ itself as the numerical result of the induced macro-magnetic field. Of course, our method can also be used with piecewise constant finite element magnetisation $\mathbf{m}$ and piecewise affine non-conforming finite element potential $u$ without any difficulty.

The rest of the paper is arranged as follows. In section 2, the method of using unknown multi-atomic points for Young measure approximation is introduced. In section 3, the method for the artificial boundary and finite element approximation of the Maxwell's equation (1.2) is given. In section 4, 
the discrete relaxed problem is formulated and analyzed. Finally, in section 5, some numerical examples are given to show the efficiency of the method where the conjugate gradient method combined with a linear search is applied to solve the discrete optimization problem.

\section{Young MEASURE APPROXIMATION}

First, we introduce some definitions and basic results (see $[7,9]$ ).

We denote $f=g \otimes \psi$ if $f(x, A)=g(x) \psi(A)$ for almost all $x \in \Omega$ and all $A \in S^{n-1}$ with $g \in L^{1}(\Omega)$ and $\psi \in C\left(S^{n-1}\right)$.

Let $\mathfrak{T}_{h_{1}}^{1}$ be a regular triangulation of $\Omega$ with mesh size $h_{1}[21]$, and let $\mathfrak{T}_{h_{2}}^{2}$ be a regular triangulation of $S^{n-1}$ with mesh size $h_{2}$. Then, $\mathfrak{T}_{h}=\mathfrak{T}_{h_{1}}^{1} \times \mathfrak{T}_{h_{2}}^{2}$ defines a triangulation on $\Omega \times S^{n-1}$ with mesh size $h=\left(h_{1}, h_{2}\right)$.

Define a projector $P_{h_{1}}^{1}: L^{1}\left(\Omega ; C\left(S^{n-1}\right)\right) \rightarrow L^{1}\left(\Omega ; C\left(S^{n-1}\right)\right)$ by

$$
\left[P_{h_{1}}^{1} f\right](x, A)=\frac{1}{K} \int_{K} f(y, A) d y, \quad \text { if } x \in K \in \mathfrak{T}_{h_{1}}^{1}
$$

and define a projector $P_{h_{2}}^{2}$ by

$$
\left[P_{h_{2}}^{2} f\right](x, A)=\sum_{i=1}^{N_{h_{2}}} f\left(x, A_{i}\right) v_{i}(A),
$$

where $v_{i}$ are element-wise affine basis functions derived from the barycentric coordinates of finite elements in $\mathfrak{T}_{h_{2}}^{2}$ (see [21]), which satisfy $v_{i}\left(A_{i}\right)=1$, $v_{i}\left(A_{j}\right)=0$ if $i \neq j$ for all $1 \leq i, j \leq N_{h_{2}}$, and $\sum_{i=1}^{N_{h_{2}}} v_{i}(A)=1$ for all $A \in S^{n-1}$. It is easily seen that $P_{h}=P_{\left(h_{1}, h_{2}\right)}=P_{h_{1}}^{1} P_{h_{2}}^{2}=P_{h_{2}}^{2} P_{h_{1}}^{1}$ defines a projector which provides an $\Omega$-element-wise constant and $S^{n-1}$-element-wise affine approximation. The adjoint projector $P_{h}^{*}: \mathfrak{A} \rightarrow \mathfrak{A}$ is defined by

$$
\left\langle P_{h}^{*} \nu, f\right\rangle=\left\langle\nu, P_{h} f\right\rangle
$$

where $\mathfrak{A}$ is defined by (1.3) and

$$
\langle\nu, f\rangle=\int_{\Omega} \int_{S^{n-1}} f(x, A) \nu_{x}(d A) d x .
$$


We denote $P_{h}^{*} \mathfrak{A} \subset \mathfrak{A}$ by $\mathfrak{A}_{h}$ (see [7] Sect. 3.5 , see also [9]). It is shown in [7] that

$$
\mathfrak{A}_{h}=\left\{\nu^{h}=\left\{\left.\nu^{h}\right|_{K}\right\}_{K \in \mathfrak{T}_{h_{1}}^{1}},\left.\nu^{h}\right|_{K}=\sum_{i=1}^{N_{h_{2}}} \lambda_{K, i} \delta_{A_{i}}, \forall K \in \mathfrak{T}_{h_{1}}^{1}\right\},
$$

where $\lambda_{K, i}$ are non-negative constants satisfying $\sum_{i=1}^{N_{h_{2}}} \lambda_{K, i}=1$ for all $K \in$ $\mathfrak{T}_{h_{1}}^{1}$, and where $\delta_{A_{i}}$ is the Dirac measure supported at $A_{i} \in S^{n-1}$.

It is also shown in [7] that

$$
\lim _{h \rightarrow 0}\left\|P_{h} f-f\right\|_{L^{1}\left(\Omega ; C\left(S^{n-1}\right)\right)}=0, \quad \forall f \in L^{1}\left(\Omega ; C\left(S^{n-1}\right)\right),
$$

and thus we have

$$
\begin{aligned}
& \quad\left|\left\langle\nu-P_{h}^{*} \nu, f\right\rangle\right|=\left|\left\langle\nu, f-P_{h} f\right\rangle\right| \\
& \quad \leq\|\nu\|_{\left(L^{1}\left(\Omega ; C\left(S^{n-1}\right)\right)\right)^{*}}\left\|f-P_{h} f\right\|_{L^{1}\left(\Omega ; C\left(S^{n-1}\right)\right)} \rightarrow 0, \text { as } h \rightarrow 0,
\end{aligned}
$$

i.e. weak $^{*}-\lim _{h \rightarrow 0} P_{h}^{*} \nu=\nu$ in $\left(L^{1}\left(\Omega ; C\left(S^{n-1}\right)\right)\right)^{*}($ see $[7,9])$.

For any $\mu^{h} \in \mathfrak{A}_{h}$, let $\left.\mathbf{m}_{h}\right|_{K \in \mathfrak{T}_{h_{1}}^{1}}=\int_{S^{n-1}} A \mu_{K}^{h}(d A)$, let $u_{\mathbf{m}_{h}}$ be the solution to the Maxwell's equation (1.2), and define

$$
R E\left(\mu^{h}\right)=\int_{\Omega} \int_{S^{n-1}} \varphi(A) \mu^{h}(d A) d x-\int_{\Omega} \mathbf{H} \cdot \mathbf{m}_{h} d x+\frac{1}{2} \int_{R^{n}}\left|\nabla u_{\mathbf{m}_{h}}\right|^{2} d x .
$$

Then, the semi-discrete Young measure version of the relaxed problem of micromagnetics is to minimize the relaxed energy $R E(\cdot)$ in the set $\mathfrak{A}_{h}$ (see [9]). By the sequential weak* compactness of $\mathfrak{A}$, the sequential weak* semicontinuity of $R E(\cdot)$ and weak* continuity of $P_{h}^{*}$, it is easily seen that the semi-discrete relaxed problem admits a solution for any $h$ with $h_{1}, h_{2}>0$ (see [9]). We also have the following convergence result for the solutions of the semi-discrete relaxed problem (see also [9])

Theorem 2.1. Let $\varphi \in C^{0}\left(S^{n-1}\right)$ and $\mathbf{H} \in L^{2}(\Omega)$. Then

$$
\lim _{h \rightarrow 0} \inf _{\mu^{h} \in \mathfrak{A}_{h}} R E\left(\mu^{h}\right)=\inf _{\mu \in \mathfrak{A}} R E(\mu) .
$$

Proof. Let $\nu \in \mathfrak{A}$ be a solution to the relaxed problem, let $\nu^{h}=P_{h}^{*} \nu \in \mathfrak{A}_{h}$ and let $\mu^{h} \in \mathfrak{A}_{h}$ be a solution to the semi-discrete relaxed problem. Then, we have

$$
0 \leq R E\left(\mu^{h}\right)-R E(\nu) \leq R E\left(\nu^{h}\right)-R E(\nu)
$$




$$
\begin{aligned}
= & \left\langle\nu^{h}-\nu, 1 \otimes \varphi\right\rangle-\left\langle\nu^{h}-\nu, \mathbf{H} \otimes \mathbf{i d}\right\rangle+\frac{1}{2}\left(\left|u_{\mathbf{m}_{h}}\right|_{1,2, R^{n}}^{2}-\left|u_{\mathbf{m}}\right|_{1,2, R^{n}}^{2}\right) \\
\leq & \left\langle\nu, P_{h}(1 \otimes \varphi-\mathbf{H} \otimes \mathbf{i d})-(1 \otimes \varphi-\mathbf{H} \otimes \mathbf{i d})\right\rangle+\left\|\mathbf{m}-\mathbf{m}_{h}\right\|_{L^{2}\left(\Omega ; R^{n}\right)} \\
\leq & \|\nu\|_{\left(L^{1}\left(\Omega ; C\left(S^{n-1}\right)\right)\right)^{*}}\left(\left\|P_{h}(1 \otimes \varphi)-1 \otimes \varphi\right\|_{L^{1}\left(\Omega ; C\left(S^{n-1}\right)\right)}\right. \\
& \left.+\left\|P_{h}(\mathbf{H} \otimes \mathbf{i d})-\mathbf{H} \otimes \mathbf{i d}\right\|_{L^{1}\left(\Omega ; C\left(S^{n-1}\right)\right)}\right)+\left\|\mathbf{m}-\mathbf{m}_{h}\right\|_{L^{2}\left(\Omega ; R^{n}\right)},
\end{aligned}
$$

where $\mathbf{m}_{h}(x)=\left.\mathbf{m}_{h}\right|_{K}$ for $x \in K \in \mathfrak{T}_{h_{1}}^{1}$ with $\left.\mathbf{m}_{h}\right|_{K}=\int_{S^{n-1}} A \nu_{K}^{h}(d A)=$ $\sum_{i=1}^{N_{h_{2}}} \lambda_{K, i}\left(\nu_{K}^{h}\right) \delta_{A_{i}}$ and $\mathbf{m}(x)=\int_{S^{n-1}} A \nu_{x}(d A)$. It follows from (2.2) that the first term on the right hand side of (2.6) converges to zero as $h \rightarrow 0$. For the second term on the right hand side of (2.6), we have

$$
\begin{aligned}
\left\|\mathbf{m}-\mathbf{m}_{\left(h_{1}, h_{2}\right)}\right\|_{L^{2}\left(\Omega ; R^{n}\right)} \leq\left\|\mathbf{m}-\mathbf{m}_{\left(h_{1}, 0\right)}\right\|_{L^{2}\left(\Omega ; R^{n}\right)}+ \\
\left\|\mathbf{m}_{\left(h_{1}, 0\right)}-\mathbf{m}_{\left(h_{1}, h_{2}\right)}\right\|_{L^{2}\left(\Omega ; R^{n}\right)},
\end{aligned}
$$

and it is easily seen that $\lim _{h_{1} \rightarrow 0}\left\|\mathbf{m}-\mathbf{m}_{\left(h_{1}, 0\right)}\right\|_{L^{2}\left(\Omega ; R^{n}\right)}=0$, hence we only need to show that the second term on the right hand side of (2.7) converges to zero as $h_{2} \rightarrow 0$.

Let $\mathbf{w} \in L^{2}\left(\Omega ; R^{n}\right)$, by the definitions, we have

$$
\begin{aligned}
& \int_{\Omega}\left(\mathbf{m}_{\left(h_{1}, h_{2}\right)}-\mathbf{m}_{\left(h_{1}, 0\right)}\right) \cdot \mathbf{w} d x=\left\langle\left(P_{\left(h_{1}, h_{2}\right)}^{*}-\left(P_{\left(h_{1}, 0\right)}^{*}\right) \nu, \mathbf{w} \otimes \mathbf{i d}\right\rangle\right. \\
= & \left\langle\nu,\left(P_{\left(h_{1}, h_{2}\right)}-\left(P_{\left(h_{1}, 0\right)}\right)(\mathbf{w} \otimes \mathbf{i d})\right\rangle=\left\langle\nu, P_{h_{1}}^{1} \mathbf{w} \otimes\left(P_{h_{2}}^{2} \mathbf{i d}-\mathbf{i d}\right)\right\rangle\right. \\
= & \sum_{K \in \mathfrak{T}_{h_{1}}^{1}} \int_{K} \int_{S^{n-1}}\left(\frac{1}{|K|} \int_{K} \mathbf{w}(y) d y\right) \cdot\left(\sum_{i=1}^{N_{h_{2}}} A_{i} v_{i}(A)-A\right) \nu_{x}(d A) d(2.8)
\end{aligned}
$$

Since $\sum_{i=1}^{N_{h_{2}}} A_{i} v_{i}(A)$ is the element-wise affine interpolation of $A$ and $\nu$ is a probability measure on $S^{n-1}$, it is easily seen that

$$
\int_{S^{n-1}}\left|\sum_{i=1}^{N_{h_{2}}} A_{i} v_{i}(A)-A\right| \nu_{x}(d A) \leq h_{2} .
$$

Therefore, by (2.8), we have

$$
\left|\int_{\Omega}\left(\mathbf{m}_{\left(h_{1}, h_{2}\right)}-\mathbf{m}_{\left(h_{1}, 0\right)}\right) \cdot \mathbf{w} d x\right| \leq\left(\int_{\Omega}|\mathbf{w}| d x\right) h_{2} \leq\|\mathbf{w}\|_{L^{2}\left(\Omega ; R^{n}\right)} \sqrt{|\Omega|} h_{2} .
$$

This leads to

$$
\left\|\mathbf{m}_{\left(h_{1}, h_{2}\right)}-\mathbf{m}_{\left(h_{1}, 0\right)}\right\|_{L^{2}\left(\Omega ; R^{n}\right)} \leq \sqrt{|\Omega|} h_{2}
$$


and the proof is complete.

Theorem 2.1 guarantees the convergence of the conforming finite element approximation for general Young measure solutions as $h \rightarrow 0$.

Remember that the Young measure $\nu$, which we want to approximate, has the property that $\nu_{x}$ is supported at most at $n+1$ points on $S^{n-1}$, and the discrete Young measure solution to the semi-discrete relaxed problem typically supported at a very few atoms (the so called active set) [9], therefore it is inefficient to work on the set $\mathfrak{A}_{h}$. In [9], an adaptive active set strategy via Weierstrass maximum principle is used to single out the active atoms. Here we provide another approach, in which only limited number of atoms $A_{K, i} \in S^{n-1}$, $1 \leq i \leq k$ are used on each element $K$ in $\mathfrak{T}_{h_{1}}^{1}$, however these atoms are a set variables instead of being fixed on the nodes of $\mathfrak{T}_{h_{2}}^{2}$. More precisely, for a given integer $k \geq 1$, we define

$$
\begin{aligned}
& \mathfrak{A}_{h_{1}}^{k}=\left\{\nu^{h_{1}, k}=\left\{\left.\nu^{h_{1}, k}\right|_{K}\right\}_{K \in \mathfrak{T}_{h_{1}}^{1}},\left.\nu^{h_{1}, k}\right|_{K}=\sum_{i=1}^{k} \lambda_{K, i} \delta_{A_{K, i}},\right. \\
&\left.A_{K, i} \in S^{n-1}, \forall 1 \leq i \leq k, \text { and } \forall K \in \mathfrak{T}_{h_{1}}^{1}\right\},
\end{aligned}
$$

where $\lambda_{K, i}$ are non-negative constants satisfying $\sum_{i=1}^{k} \lambda_{K, i}=1$ for all $K \in$ $\mathfrak{T}_{h_{1}}^{1}$, and we propose the following semi-discrete Young measure version of the relaxed problem

$(\mathbf{S D R P})$ : minimize the relaxed energy $R E(\cdot)$ in the set $\mathfrak{A}_{h_{1}}^{k}$,

where $R E(\cdot)$ is given by (2.4) with $\left.\mathbf{m}_{h}\right|_{K \in \mathfrak{T}_{h_{1}}^{1}}=\sum_{i=1}^{k} \lambda_{K, i} A_{K, i}$, and $u_{\mathbf{m}_{h}}$ being the solution to the Maxwell's equation (1.2).

Theorem 2.2. Let $\varphi \in C^{0}\left(S^{n-1}\right)$ and $\mathbf{H} \in L^{2}(\Omega)$. Then the problem (SDRP) admits a solution for any $h_{1}>0$ and $k \geq 1$.

Proof. The theorem follows from the sequential weak* compactness of $\mathfrak{A}_{h_{1}}^{k}$ and the sequential weak ${ }^{*}$ semicontinuity of $R E(\cdot)$.

Theorem 2.3. Let $\varphi \in C^{0}\left(S^{n-1}\right)$ and $\mathbf{H} \in L^{2}\left(\Omega ; R^{n}\right)$. Then

$$
\lim _{h_{1} \rightarrow 0, k \rightarrow \infty} \inf _{\mu^{h_{1}, k} \in \mathfrak{A}_{h_{1}}^{k}} R E\left(\mu^{h_{1}, k}\right)=\inf _{\mu \in \mathfrak{A}} R E(\mu) .
$$


Proof. Let $\mu^{h_{1}, k} \in \mathfrak{A}_{h_{1}}^{k}$ be a solution to (SDRP) and let $\mu^{\left(h_{1}, h_{2}\right)}$ be such that $R E\left(\mu^{\left(h_{1}, h_{2}\right)}\right)=\inf _{\nu^{\left(h_{1}, h_{2}\right)} \in \mathfrak{A}_{\left(h_{1}, h_{2}\right)}} R E\left(\nu^{\left(h_{1}, h_{2}\right)}\right)$. Since $\mathfrak{A}_{\left(h_{1}, h_{2}\right)} \subset \mathfrak{A}_{h_{1}}^{k}$ for all $k \geq N_{h_{2}}$, we have

$$
R E\left(\mu^{h_{1}, k}\right) \leq R E\left(\mu^{\left(h_{1}, h_{2}\right)}\right), \quad \forall k \geq N_{h_{2}} .
$$

This together with Theorem 2.1 proves (2.12).

Corollary 2.1. If a minimizer $\nu^{\left(h_{1}, h_{2}\right)}$ of $R E(\cdot)$ in $\mathfrak{A}_{\left(h_{1}, h_{2}\right)}$ has no more than $k_{\left(h_{1}, h_{2}\right)}$ active atoms, then we have

$$
\inf _{\mu^{h_{1}, k} \in \mathfrak{A}_{h_{1}}^{k}} R E\left(\mu^{h_{1}, k}\right) \leq \inf _{\mu^{\left(h_{1}, h_{2}\right)} \in \mathfrak{A}_{\left(h_{1}, h_{2}\right)}} R E\left(\mu^{\left(h_{1}, h_{2}\right)}\right), \quad \forall k \geq k_{\left(h_{1}, h_{2}\right)} .
$$

Proof. The relation (2.13) follows from the fact that $\nu^{\left(h_{1}, h_{2}\right)}$ is contained in the set $\mathfrak{A}_{h_{1}}^{k}$.

Since a discrete Young measure typically supported at a very few atoms, Corollary 2.1 implies that very small $k$ can be used in practical computations.

Corollary 2.2. Let $k_{\left(h_{1}, h_{2}\right)}$ be the smallest possible number of active atoms a minimizer of $R E(\cdot)$ in $\mathfrak{A}_{\left(h_{1}, h_{2}\right)}$ can have. Suppose that there exist constants $\bar{h}_{1}, \bar{h}_{2}>0$ and $k_{0} \geq 1$ such that, for any $h_{1} \in\left(0, \bar{h}_{1}\right)$ and $h_{2} \in\left(0, \bar{h}_{2}\right)$, the inequality $k_{\left(h_{1}, h_{2}\right)} \leq k_{0}$ is satisfied. Then, we have

$$
\lim _{h_{1} \rightarrow 0} \inf _{\mu^{h_{1}}, k_{0} \in \mathfrak{A}_{h_{1}}^{k_{0}}} R E\left(\mu^{h_{1}, k_{0}}\right)=\inf _{\mu \in \mathfrak{A}} R E(\mu) .
$$

Proof. The conclusion is a direct consequence of Theorem 2.1 and Corollary 2.1.

In the case when the nonconvex energy density $\varphi$ and the external magnetic field $\mathbf{H}$ are sufficiently smooth, and the solution $\mathbf{m}$ is also sufficiently smooth, the convergence rates of the semi-discrete relaxed problem can be obtained.

Corollary 2.3. Let the nonconvex energy density $\varphi \in C^{0}\left(S^{n-1}\right)$ be Lipschize continuous and the external magnetic field $\mathbf{H} \in W^{1,2}\left(\Omega ; R^{n}\right)$. Let $\nu \in \mathfrak{A}$ be a minimizer of $R E(\cdot)$ in $\mathfrak{A}$ and suppose that $\mathbf{m} \in W^{1,2}\left(\Omega ; R^{n}\right)$ where $\mathbf{m}$ is 
defined by $\mathbf{m}(x)=\int_{S^{n-1}} A \nu_{x}(d A)$. Let $\mu^{h} \in \mathfrak{A}_{h}$ be a minimizer of $R E(\cdot)$ in $\mathfrak{A}_{h}$. Then, we have

$$
\left|R E(\nu)-R E\left(\mu^{h}\right)\right|=O\left(h_{1}+h_{2}\right), \quad \forall h_{1}, h_{2}>0 .
$$

Furthermore, let $\mu^{h_{1}, k} \in \mathfrak{A}_{h_{1}}^{k}$ be a minimizer of $R E(\cdot)$ in $\mathfrak{A}_{h_{1}}^{k}$. Then, we have

$$
\left|R E(\nu)-R E\left(\mu^{h 1, k}\right)\right|=O\left(h_{1}+h_{2}\right), \quad \forall h_{1}>0, \quad k \geq k_{\left(h_{1}, h_{2}\right)} .
$$

Especially, under the assumptions of Corollary 2.2, we have

$$
\left|R E(\nu)-R E\left(\mu^{h 1, k}\right)\right|=O\left(h_{1}\right), \quad \forall h_{1}>0, \quad k \geq k_{0} .
$$

Proof. The conclusions of the corollary follows from (2.6), (2.7), (2.9), (2.13), the smoothness assumptions on $\varphi, \mathbf{H}$ and $\mathbf{m}$, and the standard finite element approximation results for functions in Sobolev spaces [21].

Remark 2.1. Regularity assumptions of $\mathbf{m} \in W^{1,2}\left(\Omega ; R^{n}\right)$ and the existence of $k_{0}$ are not known to the authors to hold in the general setting.

\section{ARTIFICIAL BOUNDARY AND FINITE ELEMENT APPROXIMATION}

In this section we condider the solution of the Maxwell's equation

$$
\begin{aligned}
& \operatorname{div}\left(-\nabla u+\mathbf{m} \chi_{\Omega}\right)=0, \quad \text { in } H^{-1}\left(R^{n}\right), \\
& u \rightarrow 0, \quad \text { as }|x| \rightarrow \infty .
\end{aligned}
$$

Let

$$
\Omega_{i}=\{x:|x|<R\}
$$

$\Omega_{e}=R^{2} \backslash \bar{\Omega}_{i}$, and

$$
\Gamma_{R}=\{x:|x|=R\}
$$

with $R$ large enough such that $\bar{\Omega} \subset \Omega_{i}$. Clearly, if $u(R, \theta) \in H^{1 / 2}\left(\Gamma_{R}\right)$ is given on the boundary $\Gamma_{R}$, then the restriction of $u$ on $\Omega_{i}$ is a solution to the following problem:

$$
\begin{aligned}
& \operatorname{div}\left(-\nabla u+\mathbf{m} \chi_{\Omega}\right)=0, \quad \text { in } \Omega_{i}, \\
& u=u(R, \theta), \quad \text { on } \Gamma_{R} .
\end{aligned}
$$


In fact, the problem (3.3)-(3.4) has a unique weak solution $u$ for any given $u(R, \theta) \in H^{1 / 2}\left(\Gamma_{R}\right)$, which defines on $\Gamma_{R}$ a function

$$
\left.\frac{\partial u}{\partial \mathbf{n}}\right|_{\Gamma_{R}} \in H^{-1 / 2}\left(\Gamma_{R}\right),
$$

Where $\mathbf{n}$ is the unit exterior normal of $\Omega_{i}$ defined on $\Gamma_{R}$.

On the other hand, we can define a bounded operator

$$
\begin{aligned}
E: & H^{1 / 2}\left(\Gamma_{R}\right) \rightarrow H^{-1 / 2}\left(\Gamma_{R}\right), \\
& \left.\frac{\partial u}{\partial \mathbf{n}}\right|_{\Gamma_{R}}=E\left(\left.u\right|_{\Gamma_{R}}\right)
\end{aligned}
$$

as follows. Consider the problem:

$$
\begin{aligned}
& -\Delta u=0, \text { in } \Omega_{e}, \\
& u=u(R, \theta), \quad \text { on } \Gamma_{R}, \\
& u \rightarrow 0 \text { as }|x| \rightarrow \infty,
\end{aligned}
$$

It is well known that the solution of this problem can be written as

$$
u(r, \theta)=\frac{a_{0}}{2}+\sum_{n=1}^{\infty}\left(\frac{R}{r}\right)\left(a_{n} \cos n \theta+b_{n} \sin n \theta\right)
$$

with

$$
a_{0}=\frac{1}{\pi} \int_{0}^{2 \pi} u(R, \phi) d \phi=0
$$

Then we have

$$
\frac{\partial u(R, \theta)}{\partial r}=\sum_{n=1}^{\infty}-\frac{n}{R}\left(a_{n} \cos n \theta+b_{n} \sin n \theta\right)
$$

and

$$
\frac{\partial^{2} u(R, \theta)}{\partial \theta^{2}}=\sum_{n=1}^{\infty}\left(-n^{2}\right)\left(a_{n} \cos n \theta+b_{n} \sin n \theta\right) .
$$

From (3.10) we obtain

$$
a_{n}=-\frac{1}{\pi n^{2}} \int_{0}^{2 \pi} \frac{\partial^{2} u(R, \phi)}{\partial \phi^{2}} \cos n \phi d \phi
$$




$$
b_{n}=-\frac{1}{\pi n^{2}} \int_{0}^{2 \pi} \frac{\partial^{2} u(R, \phi)}{\partial \phi^{2}} \sin n \phi d \phi .
$$

Therefore,

$$
\frac{\partial u(R, \theta)}{\partial \mathbf{n}}=\sum_{n=1}^{\infty} \frac{1}{\pi n R} \int_{0}^{2 \pi} \frac{\partial^{2} u(R, \phi)}{\partial \phi^{2}} \cos n(\theta-\phi) d \phi \equiv E u .
$$

Using this boundary condition we can reduce the original problem to the following equivalent problem:

$$
\begin{aligned}
& \operatorname{div}\left(-\nabla u+\mathbf{m} \chi_{\Omega}\right)=0, \quad \text { in } \Omega_{i}, \\
& \frac{\partial u}{\partial n}=E u, \quad \text { on } \Gamma_{R}, \\
& \int_{0}^{2 \pi} u(R, \phi) d \phi=0 .
\end{aligned}
$$

Let

$$
V=\left\{v \in H^{1}\left(\Omega_{i}\right): \int_{0}^{2 \pi} u(R, \phi) d \phi=0\right\} .
$$

Then (3.11)-(3.13) has the weak formulation

$$
\left\{\begin{array}{l}
\text { Find } u \in V, \text { such that } \\
a(u, v)+b(u, v)=f(v) \quad \forall v \in V
\end{array}\right.
$$

where

$$
\begin{aligned}
& a(u, v)=\int_{\Omega_{i}} \nabla u \nabla v d x, \quad f(v)=\int_{\Omega_{i}} \mathbf{m} \nabla v d x \\
& b(u, v)=\sum_{n=1}^{\infty} \frac{1}{n \pi} \int_{0}^{2 \pi} \int_{0}^{2 \pi} \cos n(\theta-\phi) \frac{\partial u(R, \theta)}{\partial \theta} \frac{\partial v(R, \phi)}{\partial \phi} d \theta d \phi .
\end{aligned}
$$

The bilinear form $a(u, v)+b(u, v)$ is symmetric, continuous, and V-elliptic on $V \times V[17]$, using Lax-Milgram theorem we have

Theorem 3.1. Problem (3.14) has a unique solution.

For the finite element approximation, let $V_{h} \subset V$ be a piecewise linear finite element subspace, we consider the approximation of problem (3.14):

$$
\left\{\begin{array}{l}
\text { Find } u_{h} \in V_{h}, \text { such that } \\
a\left(u_{h}, v_{h}\right)+b_{N}\left(u_{h}, v_{h}\right)=f\left(v_{h}\right) \quad \forall v_{h} \in V_{h},
\end{array}\right.
$$


where

$$
b_{N}\left(u_{h}, v_{h}\right)=\sum_{n=1}^{N} \frac{1}{n \pi} \int_{0}^{2 \pi} \int_{0}^{2 \pi} \cos n(\theta-\phi) \frac{\partial u_{h}(R, \theta)}{\partial \theta} \frac{\partial v_{h}(R, \phi)}{\partial \phi} d \theta d \phi .
$$

Similarly, we have

Theorem 3.2. Problem (3.15) has a unique solution.

Remark 3.1. Theorem 3.2 says that for a given discrete magnetisation $\mathbf{m}$, the finite element solution of the potential $u$ is uniquely determined by the solution of (3.15). However, since the set $\{\operatorname{div} \mathbf{m}=0$, in the discrete sense $\}$ is not empty, we can not conclude from the theorem that the coupled discrete problem has a unique solution, even when the original problem has a unique solution (for example in the case of uniaxial energy density).

By a standard argument for finite element approximations of elliptic problems [21] and by a similar argument as in [17], it is not difficult to obtain the following convergence result.

Theorem 3.3. Assume that $u \in H^{1}\left(\Omega_{i}\right) \cap H^{1 / 2}\left(\Gamma_{R}\right)$, then

$$
\left\|u-u_{h}\right\|_{1, \Omega_{i}} \leq C\left\{\inf _{v_{h} \in V_{h}}\left\|u-v_{h}\right\|_{1, \Omega_{i}}+\sup _{w_{h} \in V_{h}} \frac{b\left(u, w_{h}\right)-b_{N}\left(u, w_{h}\right)}{\left\|w_{h}\right\|_{1, \Omega_{i}}}\right\} \rightarrow 0,
$$

Furthermore, the following error estimate holds [17].

Theorem 3.4. Assume that $u \in H^{2}\left(\Omega_{i}\right) \cap H^{k-1 / 2}\left(\Gamma_{R}\right), k \geq 2$, then

$$
\left\|u-u_{h}\right\|_{1, \Omega_{i}} \leq C\left(h\|u\|_{2, \Omega_{i}}+\frac{1}{N^{k-1}}\|u\|_{k-1 / 2, \Gamma_{R}}\right) .
$$

Remark 3.2. In general, $u$ is very smooth near $\Gamma_{R}$. Thus, the second term in (3.17) is much smaller than the first term, i.e., a small $N$ would be enough for the computation. This applies also for the case when $\mathbf{m} \in L^{2}(\Omega)$.

Remark 3.3. In the convergence analysis in the next section, for the simplicity of the notations, we always assume that $N$ is sufficiently large that the error term respect to $N$ is negligible. 


\section{DiscRETE RELAXED PROBLEM AND ITS NUMERICAL ANALYSIS}

Before introduce the complete discrete relaxed problem, we first rewrite the set $\mathfrak{A}_{h_{1}}^{k}$. For simplicity, we restrict to the case $n=2$, the case $n=3$ can be discussed in a similar way.

Let $j \geq 1$ be an integer and $k=2^{j}$. Let $K \in \mathfrak{T}_{h_{1}}^{1}$ and for $i=1,2,3, \ldots, 2^{j}$ let $\theta_{K, i} \in[-\pi, \pi] /\{-\pi, \pi\}$, i.e. $-\pi$ and $\pi$ are considered to be the same point in the set, define $A\left(\theta_{K, i}\right) \in S^{1}$ by

$$
A\left(\theta_{K, i}\right)=\left(\begin{array}{c}
\cos \left(\theta_{K, i}\right) \\
\sin \left(\theta_{K, i}\right)
\end{array}\right) .
$$

For any $K \in \mathfrak{T}_{h_{1}}^{1}$, let $\alpha_{K}=\left\{\alpha_{K, l}\right\}_{l=1}^{j}$ with $\alpha_{K, l} \in[-\pi / 2, \pi / 2], l=1, \ldots, j$, and let $i=1+i_{1} 2^{0}+i_{2} 2^{1}+\ldots+i_{j} 2^{j-1}$ with $i_{l} \in\{0,1\}$ for $l=1, \ldots, j$, define

$$
\lambda\left(\alpha_{K}, i\right)=\prod_{l=1}^{j} \operatorname{cs}\left(i_{l}, \alpha_{K, l}\right)
$$

where the function $\operatorname{cs}(\xi, \beta)$ is defined by

$$
\operatorname{cs}(\xi, \beta)= \begin{cases}\cos ^{2}(\beta), & \text { if } \xi=0 \\ \sin ^{2}(\beta), & \text { if } \xi=1\end{cases}
$$

Denote $\vec{\theta}=\left\{\theta_{K, i}: K \in \mathfrak{T}_{h_{1}}^{1}, i=1, \ldots, k\right\}$ and $\vec{\alpha}=\left\{\alpha_{K}: K \in \mathfrak{T}_{h_{1}}^{1}\right\}$, it is easily verified that

$$
\begin{aligned}
& \mathfrak{A}_{h_{1}}^{k}=\mathfrak{A}_{h_{1}}^{k}(\vec{\theta}, \vec{\alpha})=\left\{\nu^{h_{1}, k}=\left\{\left.\nu^{h_{1}, k}\right|_{K}\right\}_{K \in \mathfrak{T}_{h_{1}}^{1}}\right.: \\
&\left.\left.\nu^{h_{1}, k}\right|_{K}=\sum_{i=1}^{k} \lambda\left(\alpha_{K}, i\right) \delta_{A\left(\theta_{K, i}\right)}\right\} .
\end{aligned}
$$

For any $\mu^{h_{1}, k} \in \mathfrak{A}_{h_{1}}^{k}(\vec{\theta}, \vec{\alpha})$, let $\left.\mathbf{m}_{h_{1}, k}\right|_{K \in \mathfrak{T}_{h_{1}}^{1}}=\sum_{i=1}^{k} A\left(\theta_{K, i}\right) \lambda\left(\alpha_{K}, i\right)$, let $u_{\mathbf{m}_{h_{1}, k}}^{h_{1}}$ be the finite element solution to the problem (3.15), and let $\mathbf{H}_{K}=$ $\int_{K} \mathbf{H} d x$ for all $K \in \mathfrak{T}_{h_{1}}^{1}$, define

$$
R E_{h_{1}}\left(\mu^{h_{1}, k}\right)=\sum_{K \in \mathfrak{T}_{h_{1}}^{1}} \sum_{i=1}^{k} \varphi\left(A\left(\theta_{K, i}\right)\right) \lambda\left(\alpha_{K}, i\right)|K|
$$




$$
-\sum_{K \in \mathfrak{T}_{h_{1}}^{1}} \mathbf{H}_{K} \cdot \sum_{i=1}^{k} A\left(\theta_{K, i}\right) \lambda\left(\alpha_{K}, i\right)|K|+\frac{1}{2} \int_{R^{n}}\left|\nabla u_{\mathbf{m}_{h_{1}, k}}^{h_{1}}\right|^{2} d x
$$

Remark 4.1. It is easily seen that the only difference between $R E(\cdot)$ and $R E_{h_{1}}(\cdot)$ for a discrete Young measure $\mu^{h_{1}, k} \in \mathfrak{A}_{h_{1}}^{k}(\vec{\theta}, \vec{\alpha})$ is that in the last term the solution to the Maxwell's equation is replaced by its finite element solution.

The complete discrete Young measure version of the relaxed problem can now be given as follows

$$
(\mathbf{D R P}):\left\{\begin{array}{l}
\text { minimize the discrete relaxed energy } R E_{h_{1}}(\cdot) \\
\text { in the set } \mathfrak{A}_{h_{1}}^{k}(\vec{\theta}, \vec{\alpha}) .
\end{array}\right.
$$

Theorem 4.1. Let $\varphi \in C^{0}\left(S^{n-1}\right)$ and $\mathbf{H} \in L^{2}(\Omega)$. Then, the problem (DRP) admits a solution for any $h_{1}>0$ and any $k=2^{j}$ with $j \geq 1$.

Proof. The theorem follows from the compactness of $\mathfrak{A}_{h_{1}}^{k}(\vec{\theta}, \vec{\alpha})$, the compactness of the finite element function space $\mathfrak{U}_{h_{1}}$, and the continuity of $R E_{h_{1}}(\cdot)$ in $\mathfrak{A}_{h_{1}}^{k}(\vec{\theta}, \vec{\alpha})$.

Theorem 4.2. Let $\varphi \in C^{0}\left(S^{n-1}\right)$ and $\mathbf{H} \in L^{2}\left(\Omega ; R^{n}\right)$. Let $j \geq 1$ and $k=2^{j}$. Then, we have

$$
\lim _{h_{1} \rightarrow 0, j \rightarrow \infty} \inf _{\mu^{h_{1}, k} \in \mathfrak{A}_{h_{1}}^{k}(\vec{\theta}, \vec{\alpha})} R E_{h_{1}}\left(\mu^{h_{1}, k}\right)=\inf _{\mu \in \mathfrak{A}} R E(\mu) .
$$

Proof. Let $\nu \in \mathfrak{A}$ be a minimizer of $R E(\cdot)$ in $\mathfrak{A}$, let $\nu^{\left(h_{1}, h_{2}\right)}=P_{\left(h_{1}, h_{2}\right)}^{*} \nu$, and let $\nu^{h_{1}, k} \in \mathfrak{A}_{h_{1}}^{k}$ be a minimizer of $R E(\cdot)$ in $\mathfrak{A}_{h_{1}}^{k}$. Denote $\mathbf{m}=\int_{S^{n-1}} A \nu(d A)$ and $\tilde{\mathbf{m}}_{h}=\int_{S^{n-1}} A \nu^{\left(h_{1}, h_{2}\right)}(d A)$. Then, we have

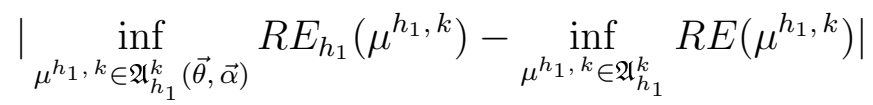

$$
\begin{aligned}
& \leq\left|R E_{h_{1}}\left(\nu^{\left(h_{1}, h_{2}\right)}\right)-R E\left(\nu^{\left(h_{1}, h_{2}\right)}\right)\right| \\
& +\left|R E\left(\nu^{\left(h_{1}, h_{2}\right)}\right)-R E(\nu)\right|+\left|R E(\nu)-R E\left(\nu^{h_{1}, k}\right)\right| \\
& \leq \frac{1}{2}\left|\int_{R^{n}}\left(\left|\nabla u_{\tilde{\mathbf{m}}_{h}}^{h_{1}}\right|^{2}-\left|\nabla u_{\tilde{\mathbf{m}}_{h}}\right|^{2}\right) d x\right|+I_{1}+I_{2},
\end{aligned}
$$


It follows from the continuity of $R E(\cdot)$ and $P_{\left(h_{1}, h_{2}\right)}^{*}$, that $I_{1}$ converges to zero as $h=\left(h_{1}, h_{2}\right) \rightarrow 0$. $I_{2}$ converges to zero is a consequence of Theorem 2.3. For the first term on the right hand side of (4.8), we have

$$
\begin{aligned}
& \left|\int_{R^{n}}\left(\left|\nabla u_{\tilde{\mathbf{m}}_{h}}^{h_{1}}\right|^{2}-\left|\nabla u_{\tilde{\mathbf{m}}_{h}}\right|^{2}\right) d x\right| \leq\left|\int_{R^{n}}\left(\left|\nabla u_{\tilde{\mathbf{m}}_{h}}^{h_{1}}\right|^{2}-\left|\nabla u_{\mathbf{m}}^{h_{1}}\right|^{2}\right) d x\right| \\
& \quad+\left|\int_{R^{n}}\left(\left|\nabla u_{\mathbf{m}}^{h_{1}}\right|^{2}-\left|\nabla u_{\mathbf{m}}\right|^{2}\right) d x\right|+\left|\int_{R^{n}}\left(\left|\nabla u_{\mathbf{m}}\right|^{2}-\left|\nabla u_{\tilde{\mathbf{m}}_{h}}\right|^{2}\right) d x\right| \\
& \leq 2\left\|\tilde{\mathbf{m}}_{h}-\mathbf{m}\right\|_{0,2, \Omega}^{2}+\left|\int_{R^{n}}\left(\left|\nabla u_{\mathbf{m}}^{h_{1}}\right|^{2}-\left|\nabla u_{\mathbf{m}}\right|^{2}\right) d x\right| .
\end{aligned}
$$

Thus, the theorem is proved by the continuity of $P_{\left(h_{1}, h_{2}\right)}^{*}$ and Theorem 3.3.

Corollary 4.1. Let $\varphi \in C^{0}\left(S^{n-1}\right)$ and $\mathbf{H} \in L^{2}\left(\Omega ; R^{n}\right)$. Let $k_{\left(h_{1}, h_{2}\right)}$ be defined as in Corollary 2.2. Suppose that there exist constants $\bar{h}_{1}, \bar{h}_{2}>0$ and $k_{0} \geq 1$ such that, for any $h_{1} \in\left(0, \bar{h}_{1}\right)$ and $h_{2} \in\left(0, \bar{h}_{2}\right)$, the inequality $k_{\left(h_{1}, h_{2}\right)} \leq k_{0}$ is satisfied. Let $j_{0} \geq 1$ and $k=2^{j_{0}} \geq k_{0}$. Then, we have

$$
\lim _{h_{1} \rightarrow 0} \inf _{\mu^{h_{1}, k} \in \mathfrak{A}_{h_{1}}^{k}(\vec{\theta}, \vec{\alpha})} R E_{h_{1}}\left(\mu^{h_{1}, k}\right)=\inf _{\mu \in \mathfrak{A}} R E(\mu) .
$$

Proof. The theorem follows directly from the inequality (4.8), Corollary 2.2 and Theorem 3.3.

Corollary 4.2. Let the nonconvex energy density $\varphi \in C^{0}\left(S^{n-1}\right)$ be Lipschize continuous and the external magnetic field $\mathbf{H} \in W^{1,2}\left(\Omega ; R^{n}\right)$. Let $\nu \in \mathfrak{A}$ be a minimizer of $R E(\cdot)$ in $\mathfrak{A}$ and suppose that $\mathbf{m} \in W^{1,2}\left(\Omega ; R^{n}\right)$ where $\mathbf{m}$ is defined by $\mathbf{m}(x)=\int_{S^{n-1}} A \nu_{x}(d A)$. Let $j \geq 1$ and $k=2^{j}$, and let $\mu^{h_{1}, k} \in \mathfrak{A}_{h_{1}}^{k}(\vec{\theta}, \vec{\alpha})$ be a minimizer of $R E_{h_{1}}(\cdot)$ in $\mathfrak{A}_{h_{1}}^{k}(\vec{\theta}, \vec{\alpha})$. Let $k_{\left(h_{1}, h_{2}\right)}$ be defined as in Corollary 2.2. Then, we have

$$
\left|R E(\nu)-R E_{h_{1}}\left(\mu^{h 1, k}\right)\right|=O\left(h_{1}+h_{2}\right), \quad \forall h_{1}>0, \quad k=2^{j} \geq k_{\left(h_{1}, h_{2}\right)} .
$$

Furthermore, under the assumptions of Corollary 4.1, we have

$$
\left|R E(\nu)-R E_{h_{1}}\left(\mu^{h 1, k}\right)\right|=O\left(h_{1}\right), \quad \forall h_{1}>0, \quad k=2^{j} \geq k_{0} .
$$

Proof. The inequality (4.8) together with Corollary 2.3 and Theorem 3.4 leads to the conclusion. 
Remark 4.2. Since any function $\mu^{h 1, k} \in \mathfrak{A}_{h_{1}}^{k}(\vec{\theta}, \vec{\alpha})$ is a function of the variables $(\vec{\theta}, \vec{\alpha})$, the functions $\left.\mathbf{m}_{h_{1}, k}\right|_{K \in \mathfrak{T}_{h_{1}}^{1}}=\sum_{i=1}^{k} A\left(\theta_{K, i}\right) \lambda\left(\alpha_{K}, i\right)$ and the finite element solution $u_{\mathbf{m}_{h_{1}, k}}^{h_{1}}$ are also functions of $(\vec{\theta}, \vec{\alpha})$. Therefore, the discrete relaxed problem (DRP) is a finite dimensional nonconvex unconstrained optimization problem defined on $\left(S^{n-1}\right)^{k} \times\left(S^{1}\right)^{j}$ with $k=2^{j}$. In computations, we may simply take $(\vec{\theta}, \vec{\alpha}) \in R^{k+j}$.

Remark 4.3. In the definition of (DRP), $k=2^{j}$ can vary from element to element, the number can be adjusted according to the number of active atoms obtained in the computation.

\section{Numerical EXAmples}

To solve the discrete relaxed problem (DRP) (4.6), which is a unconstrained nonconvex optimization problem of finite dimension, we apply the following algorithm:

(1): set $j=j_{0} \geq 1$, set $k=2^{j}$ and set $h_{1}=h_{1}^{0}$;

(2): $\operatorname{set}(\vec{\theta}, \vec{\alpha})=\left(\vec{\theta}_{0}, \vec{\alpha}_{0}\right)$;

(3): compute $R E_{h_{1}}\left(\mu^{h_{1}, k}(\vec{\theta}, \vec{\alpha})\right)$ by (4.1) - (4.3) and by solving (3.15);

(4): compute $d(\vec{\theta}, \vec{\alpha})=\frac{\partial R E_{h_{1}}\left(\mu^{h_{1}, k}(\vec{\theta}, \vec{\alpha})\right)}{\partial(\vec{\theta}, \vec{\alpha})}$;

(5): if $\|d(\vec{\theta}, \vec{\alpha})\|<$ TOL, go to step (7);

(6): search for a minimizer $\left(\vec{\theta}_{1}, \vec{\alpha}_{1}\right)$ of $R E_{h_{1}}$ along the conjugate gradient direction;

(7): if $j$ is not sufficiently large, then set $j=j+1$ and $k=2^{j}$, distribute the new atoms accordingly, then go to step (3);

(8): if $h_{1}$ is not sufficiently small, set $h_{1}=h_{1} / 2$, TOL $=\mathrm{TOL} / 2$, and initiate the data on the fine mesh, then go to step (3).

We notice here that the problem (3.15) is in fact a system of linear equations of the form

$$
K u_{\mathbf{m}}=G \mathbf{m}
$$


where $K$ is symmetric, and thus we have

$$
\int_{R^{2}}\left|\nabla u_{\mathbf{m}}\right|^{2} d x=\int_{\Omega} \mathbf{m} \cdot \nabla u_{\mathbf{m}} d x=u_{\mathbf{m}}^{T} K u_{\mathbf{m}}=\mathbf{m}^{T} G^{T} K^{-1} G \mathbf{m},
$$

and

$$
\frac{\partial \int_{R^{2}}\left|\nabla u_{\mathbf{m}}\right|^{2} d x}{\partial \mathbf{m}}=2 G^{T} K^{-1} G \mathbf{m}
$$

In the steps (3) and (4) of the algorithm, the equations (5.2) and (5.3) are used to compute the corresponding terms.

The criteria for enlarging $j$ in step (6) and reducing $h_{1}$ in step (7) may depend on the problem we solve. In general, we may enlarge $j$ on an element, if the number of active atoms is greater than $2^{j-1}$, and we may reduce $h_{1}$ if $u^{h_{1}}-u^{h_{1} / 2}$ is not sufficiently small.

To reduce the computational work, we may first replace the artificial boundary condition (3.12) and (3.13) by a Dirichlet boundary condition

$$
u_{\mathbf{m}}=0, \quad \text { on } \Gamma_{R} \text {. }
$$

and use the numerical result so obtained to provide an initial data for the problem using artificial boundary condition. Our numerical experiments show that this works well.

As mentioned in section 1, to suppress the artificial oscillations of the numerical result of $\mathbf{m}$, an additional energy term

$$
\lambda \int_{\Omega}|\mathbf{m}-\mathbf{H}|^{2} d x
$$

is added to the energy functional. Our numerical experiments show that this works well and improves the convergence behavior of the algorithm.

In the following, we are going to show some numerical examples.

Example 1. Let $\Omega=(-0.1,0.1) \times(-0.5,0.5)$ and $\Omega_{i}=\left\{x \in R^{2}:|x|<1\right\}$, that is we take $R=1$. We take $\varphi(\mathbf{m})=10^{-2}\left(m_{1}^{2}+\left(m_{2}^{2}-1\right)^{2}\right)$ and $\mathbf{H}=$ $\left(10^{-2}, 0\right)$. We set $j_{0}=1$ and TOL $=10^{-10}$. For the approximation of artificial boundary, we take $N=5$ for a coarse mesh and $N=9$ for a refined mesh. $\lambda$ is initially set to 0.01 and reduced to 0.002 in the end.

Numerical experiments show that an exactly same element-wise two-atomic Young measure is obtained whether $j$ is set to be 1,2 or 3 . 
Figure 1 shows the potential $u_{\mathbf{m}}$ obtained on the coarse mesh, and Figure 2 shows the potential obtained on the refined mesh. It is clearly seen that for the potential, there is no need to refine the mesh anymore. As a comparison, we show in Figure 3 the potential obtained on the coarse mesh for the problem with Dirichlet boundary condition (5.4). In Figure 4, we show the distribution of $\mathbf{m}$ on $\Omega$ (on the left sub-plot) and the atomic Young measure (on the right sub-plot), where two vectors in $S^{1}$ and their volume fractions, which are close to $1 / 2$, on some selected elements are shown. In Figure 4 , the number pair $(i, j)$ indicates that the corresponding Young measure is for the element respect to the vector of the $i$-th row and $j$-th column in the left hand side sub-plot.

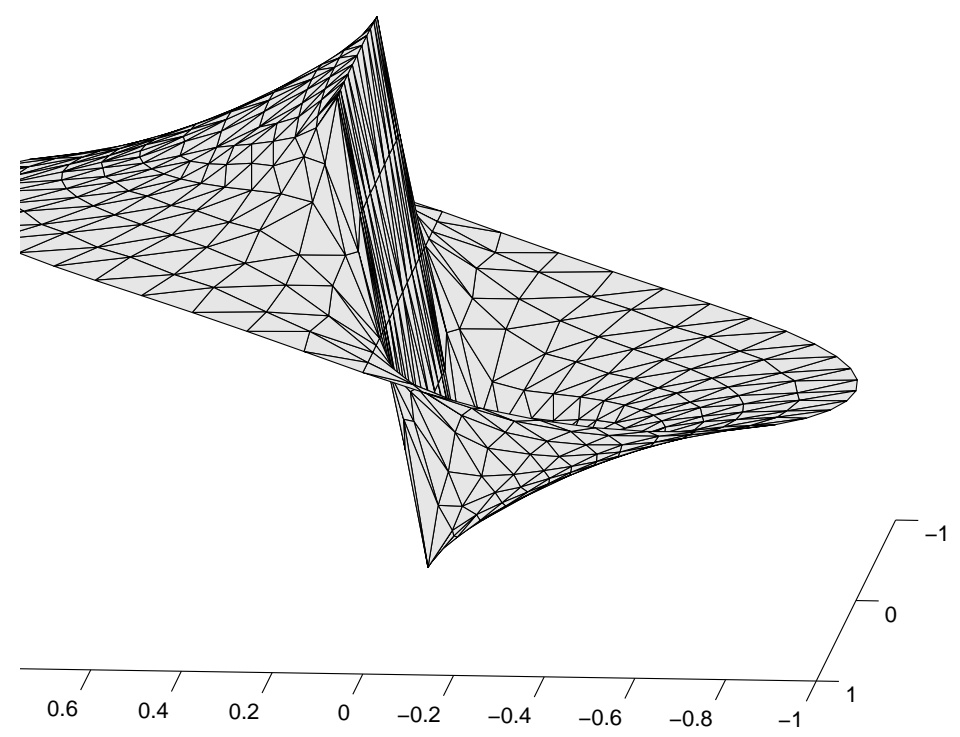

Figure 1. The potential obtained on the coarse mesh.

We point out here that our numerical experiments show that, even though most of the oscillations (microstructure) are captured by the atomic Young measures obtained on the triangular finite elements, there are still some artificial oscillations, which are discrete divergence free only in the interior of $\Omega$ and can not be suppressed by the additional energy term, show up in the element level. However, since these oscillations are in the form of pairs in neighboring elements, they can be easily removed from the numerical result by taking local averages. In Figure 4 (as well as in other relevant figures below), the induced 


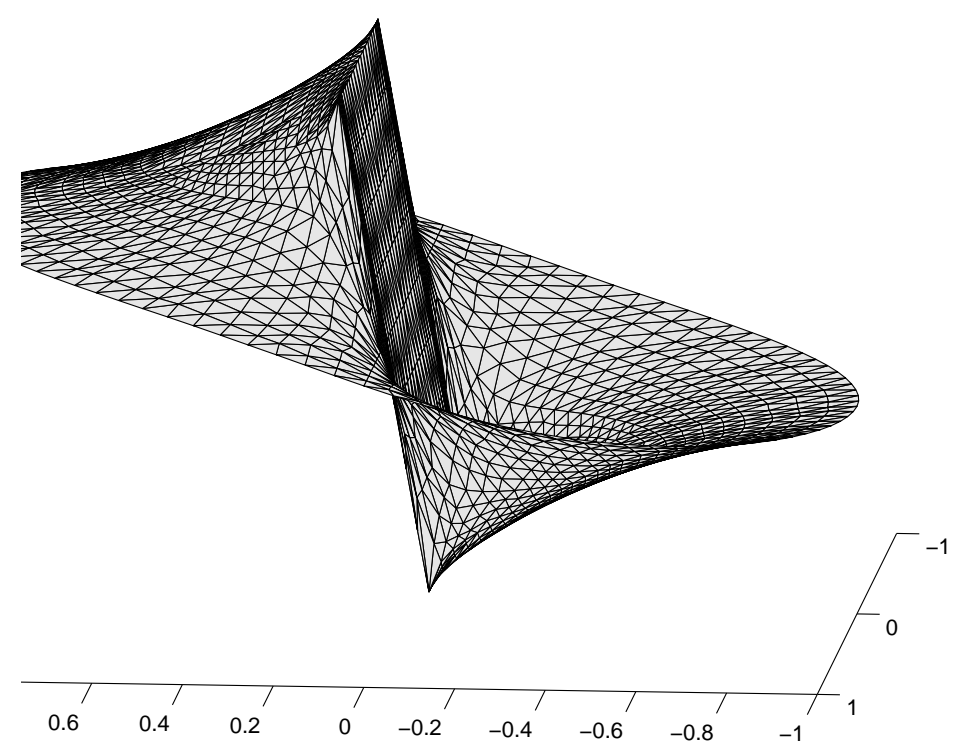

Figure 2. The potential obtained on the refined mesh.

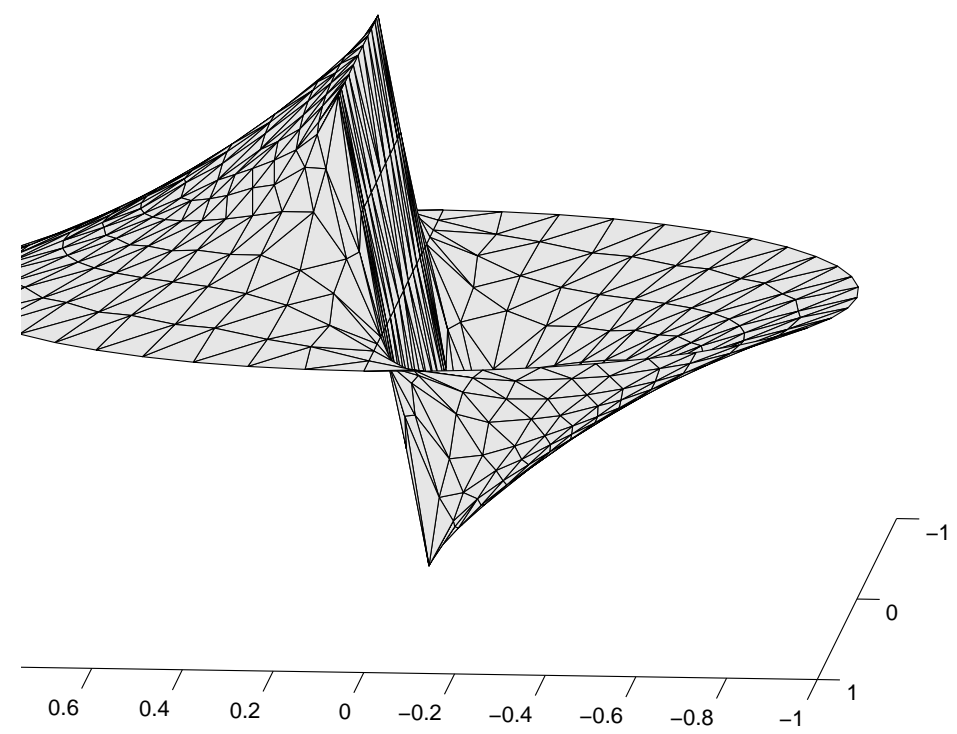

FIGURE 3. The potential with Dirichlet boundary condition obtained on the coarse mesh. 

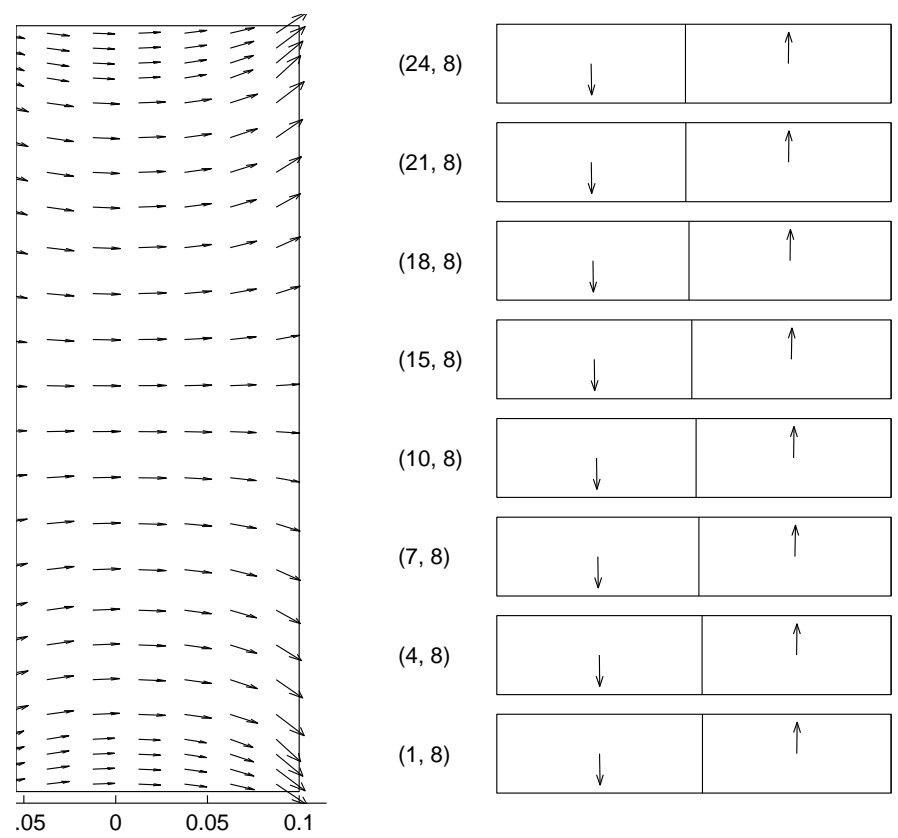

$(7,8)$

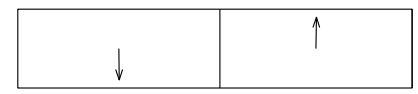

$(4,8)$

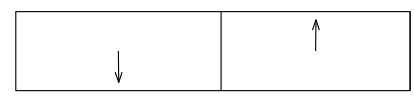

$(1,8)$

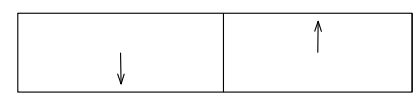

FiguRE 4. Distribution of $\mathbf{m}$ (left) and atomic Young measure (right)

magnetic vector field $\mathbf{m}$ is created on a rectangular mesh, which is obtained by combining each pair of adjacent triangular elements of the triangulation of $\Omega$, by taking the average values of $\mathbf{m}$ on the pairs of triangular elements. This shows a smoother induced macroscopic magnetic vector field.

Example 2. Again, let $\Omega=(-0.1,0.1) \times(-0.5,0.5)$ and $\Omega_{i}=\left\{x \in R^{2}\right.$ : $|x|<1\}$. We still take $\varphi(\mathbf{m})=10^{-2}\left(m_{1}^{2}+\left(m_{2}^{2}-1\right)^{2}\right)$, but take a different exterior magnetic field $\mathbf{H}=10^{-2}(\cos (y \pi) \sin (2.5 x \pi), \sin (y \pi) \cos (2.5 x \pi))$. We set $j_{0}=1$ and TOL $=10^{-10}$. Again, for the approximation of artificial boundary, we take $N=5$ for a coarse mesh and $N=9$ for a refined mesh. $\lambda$ is initially set to 0.01 and reduced to 0.002 in the end.

Figure 5 shows the potential $u_{\mathbf{m}}$ obtained on the coarse mesh, and Figure 6 shows the potential obtained on the refined mesh. As a comparison, we show in Figure 7 the potential obtained on the refined mesh for the problem with Dirichlet boundary condition (5.4). In Figure 8, we show the distribution of $\mathbf{m}$ on $\Omega$ (on the left sub-plot) and the atomic Young measure (on the right sub-plot), where two vectors in $S^{1}$ and their volume fractions, which vary from $1 / 6$ to $5 / 6$, on some selected elements are shown. 


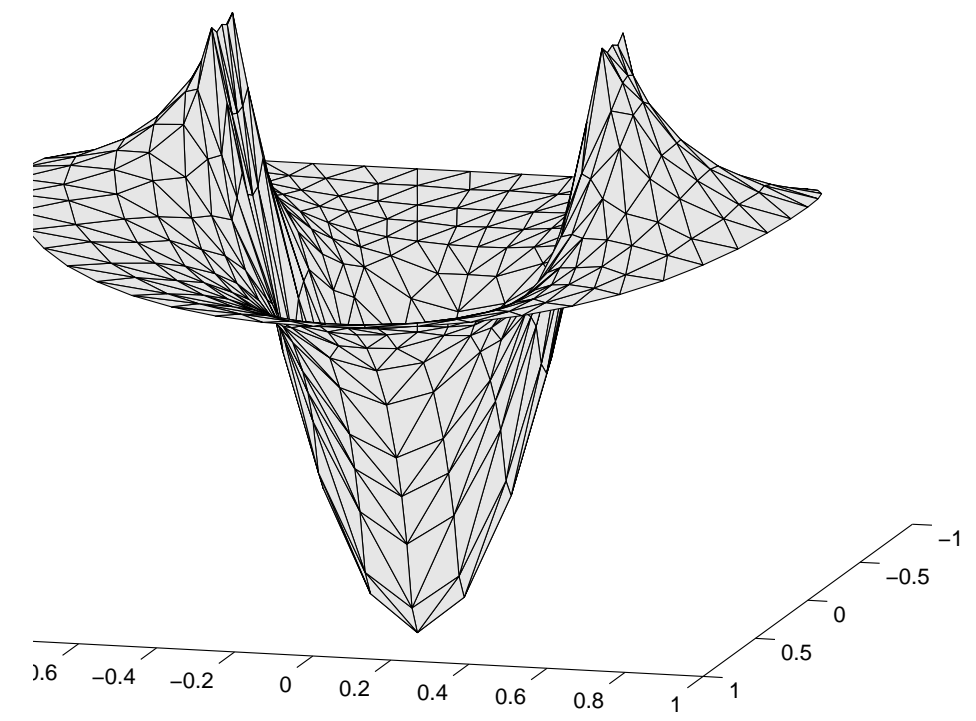

Figure 5. The potential obtained on the coarse mesh for example 2.

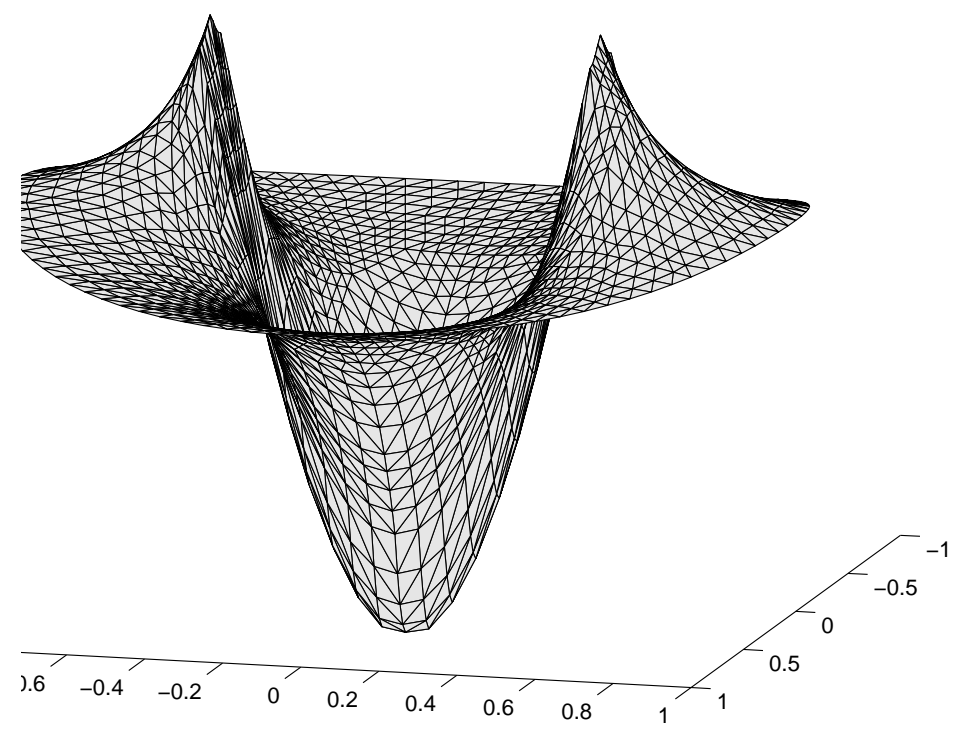

Figure 6 . The potential obtained on the refined mesh for example 2. 


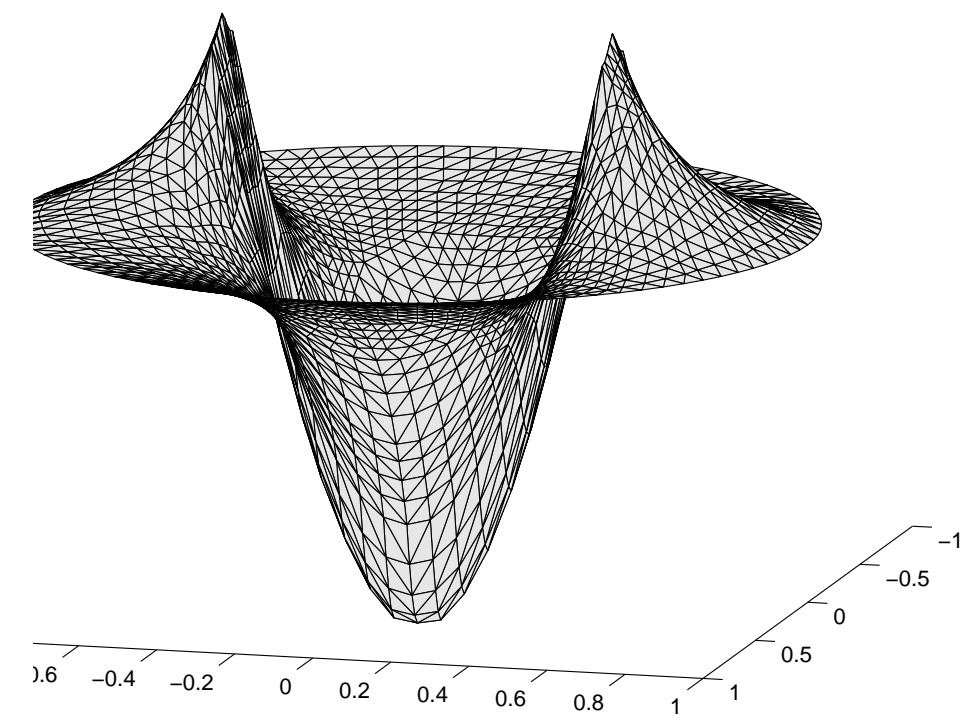

Figure 7 . The potential with Dirichlet boundary condition obtained on the refined mesh for example 2.
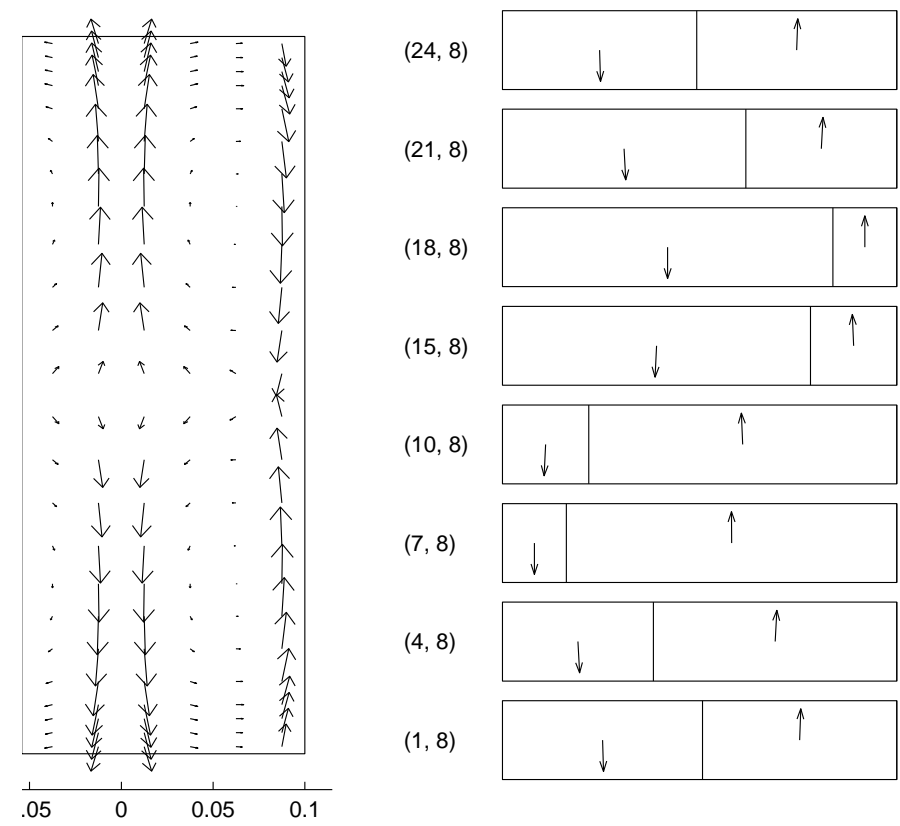

Figure 8. Distribution of $\mathbf{m}$ (left) and atomic Young measure (right) for example 2 
Remark 5.1. In our numerical experiments, we found that for the anisotropic energy density $\varphi(\mathbf{m})=10^{-2}\left(m_{1}^{2}+\left(m_{2}^{2}-1\right)^{2}\right)$ and various exterior magnetic field $\mathbf{H}$, the Young measure obtained is always supported on two atoms regardless of $j$ taking to be $1,2,3, \ldots$ This indicates that for such problems $j=1$ is sufficient in computation.

Example 3. Let $\varphi(\mathbf{m})=10^{-2}\left(m_{1}^{2} \cdot m_{2}^{2}\right)$, that is the anisotropic energy density is cubic. Let everything else be the same as in example 1 .

For all $j \geq 1$ the numerical results on the potential $u_{\mathbf{m}}$ are very close to each other and appear to be close to those shown in Figure 1-Figure 3 and the distribution of the macroscopic induced magnetic field $\mathbf{m}$ differs only slightly from that shown in Figure 4 especially for $j=1$. However, while a 2-atomic Young measure similar to that obtained in example 1 shown in Figure 4 is obtained for $j=1$, a Young measure of up to 4 atoms on each element is obtained when $j \geq 2$ is taken (see Figure 9).
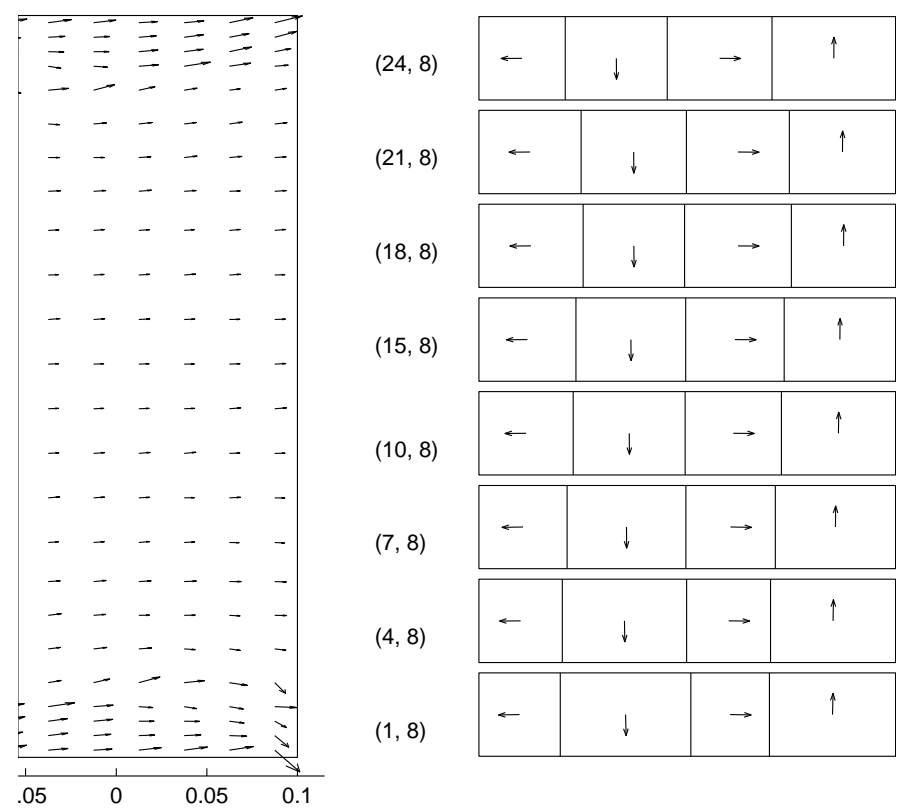

Figure 9. Distribution of $\mathbf{m}$ (left) and 4-atomic Young measure (right) for example 3

Example 4. Let $\varphi(\mathbf{m})=10^{-2}\left(m_{1}^{2} \cdot m_{2}^{2}\right)$, that is the anisotropic energy density is cubic as in example 3 , and let everything else be the same as in example 2 . 
For all $j \geq 1$ the numerical results on the potential $u_{\mathbf{m}}$ are similar to those obtained for example 2 shown in Figure 5-Figure 7. As in example 3, a 2-atomic Young measure is obtained for $j=1$, and a Young measure of up to 4 atoms is obtained for $j \geq 2$. Figure 10 shows numerical results for the distribution of the induced macroscopic magnetic field $\mathbf{m}$ and a Young measure of up to 4 atoms on each element.
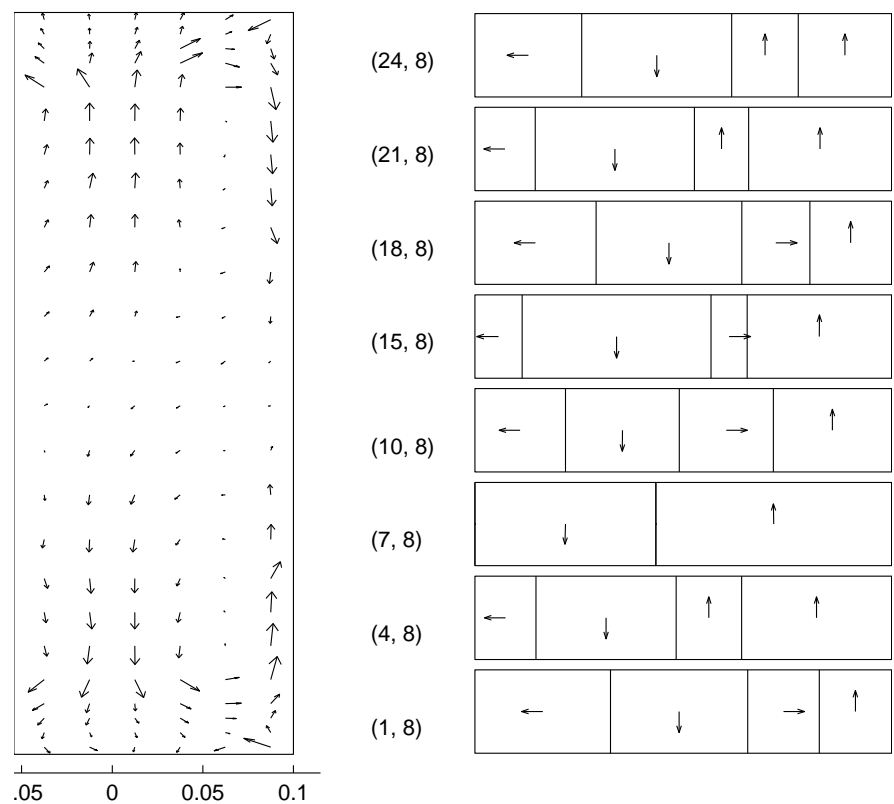

FiguRE 10. Distribution of $\mathbf{m}$ (left) and 4-atomic Young measure (right) for example 4

The numerical experiments on example 3 and example 4 show that, for 2-dimensional cubic anisotropic energy, it is natural to take $j=2$, that is to take 4-atomic Young measure as the candidates for the numerical computation of micromagnrtics.

Acknowledgment: The work was done when $\mathrm{Z}$. Li visited X. Wu at the Hong Kong Baptist University.

\section{REFERENCES}

[1] W.F. Brown, Jr., Micromagnetics. Interscience, New York, 1963.

[2] W.F. Brown, Jr., Magnetostatic Principles in Ferromagnetism. Springer, New York, 1966.

[3] R.D. James and D. Kinderlehrer, Frustration in ferromagnetic materials. Continuum Mech. Thermodym., 2(1990), 215-239. 
[4] B. Dacorogna, Direct Methods in the Calculus of Variations. Springer-Verlag, Heidelberg, 1989.

[5] P. Pedregal, Relaxation in ferromagnetism: the rigit case. J. Nonlinear Science, 4(1994), 105-125.

[6] P. Pedregal, Parameterized Measures and Variational Principles. Birkhäuser, 1997.

[7] T. Roubíček, Relaxation in Optimization Theory and Variational Calculus. W. de Gruyter, Berlin-New York, 1997.

[8] L. Tartar, Beyond Young measures. Meccanica, 30(1995), 505-526.

[9] M. Kružík and A. Prohl, Young measure approximation in micromagnetics. Numer. Math., 90(2001), 291-307.

[10] M. Kružík, Numerical solution to relaxed problem in micromagnetics. Preprint, 1997.

[11] D. Gilbarg and N.S.Trudinger, Elliptic Partial Differential Equations of the Second Order, 2nd ed.. Springer, New York, 1983.

[12] Z. Li, Rotational transformation method and some numerical techniques for the computation of microstructures. Math. Models Meth. Appl. Sci., 8(1998), 985-1002.

[13] Z. Li, A periodic relaxation method for computing microstructures. Appl. Numer. Math., 32(2000), 291-303.

[14] Z. Li, A mesh transformation method for computing microstructures. Numer. Math., 89(2001), pp. 511-533.

[15] Z. Li, Finite order rank-one convex envelopes and computation of microstructures with laminates in laminates. BIT Numer. Math., 40(4)(2000), pp.745-761.

[16] A. Bayliss, M. Gunzburger and E. Turkel, Boundary conditions for the numerical solution of elliptic equations in exterior regions. SIAM J. Appl. Math., 42(1982), 430-451.

[17] H. Han and X. Wu, Approximation of infinite boundary condition and its application to finite element methods. J. Comp. Math., 3(1985), 179-192.

[18] I. Babuška and H.S. Oh, The p-version of the finite element method for domains with corners and for infinite domains. Numer. Methods Partial Differential Equations, 6(1990), 371-392.

[19] D. Givoli, Numerical Methods for Problems in Infinite Domains. Elsevier, Amsterdam, 1992.

[20] H. Han, C. He and X. Wu, Analysis of artificial boundary conditions for exterior boundary value problems in three dimentions. Numer. Math., 85(2000), 367-386.

[21] P.G. Ciarlet, The Finite Element Method for Elliptic Problems. North-Holland, Amsterdam, 1978.

[22] C. Carstensen and A. Prohl, Numerical analysis of relaxed micromagnetics by penalised finite elements. Numer. Math., 90(2001), 65-99.

E-mail address: zpli@math.pku.edu.cn, xwu@hkbu.edu.hk 